\title{
Positive solutions for fractional boundary value problems under a generalized fractional operator
}

\author{
Mohammed S. Abdo ${ }^{1}$, Mdi Jeelani², ABDULKAFI SAEED ${ }^{3}$, and Kamal Shah ${ }^{4}$ \\ ${ }^{1}$ Hodeidah University \\ ${ }^{2}$ Imam Muhammad Ibn Saud Islamic University \\ ${ }^{3}$ Qassim University \\ ${ }^{4}$ University of Malakand
}

June 12,2020

\begin{abstract}
The work reported here concerns with study a generalized nonlinear fractional boundary value problems involving $\$ \backslash$ vartheta \$- fractional derivative in the Riemann-Liouville sense. The existence and uniqueness of positive solutions to the problem at hand are proved. Our discussion relies on the properties of the Green's function, the upper and lower solutions method, and the classical fixed point theorems in a cone. Moreover, building upper and lower control functions have an effective role in the analysis. Some examples are offered to justify the validity of theoretical findings.
\end{abstract}

\section{Hosted file}

Positive solutions on psi-RL.pdf available at https://authorea.com/users/332784/articles/ 459175-positive-solutions-for-fractional-boundary-value-problems-under-a-generalizedfractional-operator 